\title{
Knowledge of correct and incorrect items in verbal discrimination'
}

EUGENE A. LOVELACE

UNIVERSITY OF VIRGINIA

This study shows that Ss learn more about correct than incorrect members during acquisition of a 2-choice verbaldiscrimination (V-D) task. The degree of this superiority of knowledge of correct items does not seem to be related to the type of verbal KOR Ss receive ( $\mathrm{RW}, \mathrm{Rb}$, or $\mathrm{Wb}$ ), at least when KOR itself does not produce differential rates of $V-D$ list ac quisition.

The presentation of verbal knowledge of results (KOR) in 2-choice verbal-discrimination (V-D) tasks has generally taken one of three forms: (1) the Ss are told either "Right" or "Wrong" after each response (Condition $\mathrm{RW}$ ), (2) the Ss are only told when they are "Right" and E says nothing (blank) if they are wrong (Condition Rb), or (3) the Ss are told when "Wrong" but $\mathrm{E}$ says nothing if they are right (Condition $\mathrm{Wb}$ ). In a number of situations, including V-D learning, it has been reported that Ss learn slower under Condition $R b$ than either Condition $\mathrm{RW}$ or Condition $\mathrm{Wb}$, and that the latter two conditions do not differ from each other (e.g., Buchwald, 1959; Spence, 1964). In these studies Ss were not told the form that KOR would take, only that it would become apparent as they went along which were the correct responses. If the instructions make explicit the meaning or information value of $E$ saying nothing the three KOR conditions produce equal performance (Spence, Lair, \& Goodstein, 1963).

The present investigation was designed to assess what the $S$ learns about correct and incorrect items in a V-D list independent of their relation to each other, i.e., can $\mathrm{S}$ recognize correct items and incorrect items as such? Furthermore, it was of interest to see if knowledge of the two types of items (correct and incorrect) varied with the verbal KOR conditions under which the $\mathrm{S}$ learned the V-D task.

What $S$ had learned about correct and incorrect items during V-D acquisition was assessed in the present study by the use of a transfer task similar to that devised by Mcclelland (1942); i.e., the transfer list was derived from the original V-D list by replacing the incorrect member of half the pairs, and the correct member of the remaining half, with new words. The Ss in the present investigation were carried to perfect performance and then fully informed of the nature of the transfer list. Their performance on the transfer list could only reflect what had been learned during the original V-D acquisition since no KOR was given on the transfer task.

Superior performance was predicted for pairs in which the correct item was brought forward to the transfer task. It was of further interest in the present study to ascertain whether any superiority of this sort would be affected by the kind of verbal KOR which $S$ received during V-D acquisition. It was felt that when Ss gave items which were followed by a verbal response from $\mathbf{E}$ these items may have been emphasized for the $S$ such that he would learn these items and their designation ("Right" or "Wrong") better than items followed by nothing.

Method

All Ss received the same two 2-choice V-D lists. The research design was a 3 by 2 factorial with the type of KOR on the first list a between-Ss variable and member of each pair brought forward (correct vs. incorrect) a within-Ss variable. The transfer list was derived from the original $V-D$ list by replacing the correct member of half the pairs and the incorrect member of the remaining half. Pairs in the transfer task where correct members were retained will be called Sublist C; those where the incorrects were retained, Sublist I. The same word was changed in each pair for all Ss; for half the Ss this word had been correct, for half incorrect.

The 60 male college students serving as Ss were naive regarding the procedures employed in this experiment. The Ss were assigned randomly to condition, $20 \mathrm{Ss}$ per condition.

The stimuli were 36 common English words of AA frequency in Thorndike-Lorge (1944). Twenty-four were randomly paired to form the 12-pair list; the remaining words were used as the replacements in the transfer task. The words were presented at a 2-sec. rate on a memory drum.

Instructions for the V-D acquisition did not make explicit how Ss would receive KOR, only that "it will become clear to you as we go along which word in each pair is correct." Four different orders of the 12 pairs were employed. The correct member of each pair appeared half the time on the right and half on the left. Upon reaching an errorless trial on the V-D list all Ss were instructed, in part, as follows regarding the V-D transfer task: "One member of each of these pairs is a word from the list you have just learned. If that word was correct in the previous list it will remain correct in this second list. If it was incorrect before it will remain incorrect. The other word in each pair will be a new word which you have not seen before."

With each $S$ having been informed that $E$ would not give any KOR on this task, performance was recorded for four transfer trials. 


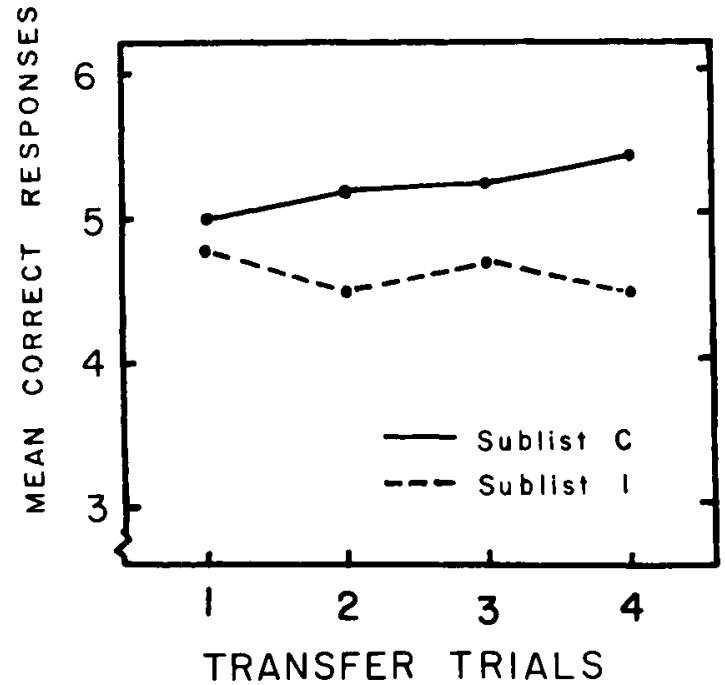

Fig. 1. Transfer list performance for pairs in which the correct (Sublist $\mathbf{C}$ ) or the incorrect (Sublist $\mathbf{I}$ ) member was retained.

\section{Results}

The mean numbers of trials to criterion on the original V-D list were 9.7, 10.6 and 11.6 for conditions $\mathrm{Wb}, \mathrm{RW}$, and $\mathrm{Rb}$, respectively. These means did not differ significantly $(\mathrm{F}<1.0)$.

A mixed factorial analysis of variance was performed on the mean numbers of correct responses on the transfer task. Transfer sublist was a highly significant source of variance $(F=15.60, p<.001)$. This resulted from performance on Sublist $\mathrm{C}$ being superior to that on Sublist I (see Fig. 1). Main effects of trials and KOR conditions yielded $F$ ratios less than 1.0. Transfer sublist was found to interact significantly with trials $(\mathrm{F}=5.94, \mathrm{p}<.001)$.

\section{Discussion}

The failure of the three KOR conditions to affect the rate of original V-D acquisition was an unexpected outcome. This suggests that the $\mathrm{Ss}$ in $\mathrm{Rb}$ and $\mathrm{Wb}$ properly deduced the meaning of $E$ saying nothing. More recent data (Spence, in press) indicates that the $\mathbf{S}$ population may determine this, with college students often doing so, but younger or less bright Ss less likely to make the proper inferences.

The superiority of performance on Sublist $C$ over Sublist I confirms Mcclelland's (1942) finding and suggests that Ss learn more about correct than incorrect items during V-D learning. The absence of an interaction of sublist with KOR conditions indicates that the amount that $S$ learns about correct and incor- rect items is not affected by the type of verbal KOR he receives. Note again, however, that no differences in original $\mathrm{V}-\mathrm{D}$ acquisition were obtained as a function of type of KOR.

The significant interaction of sublist with trials results from performance improvement across trials for pairs in Sublist $C$ while performance declines with trials on pairs of Sublist I. Presumably Ss have two tasks during the transfer portion of the present study: (1) Make an old vs, new discrimination for each pair, and (2) decide if the old member was correct or incorrect. The old-new discrimination is most likely based on "frequency of occurrence" in the experimental situation. It has been proposed (Underwood, Jesse, \& Ekstrand, 1964) that an item receives an increment in frequency each time it is seen or said by $\mathrm{S}$. For both sublists there should be an initial frequency difference between the two members of a pair. For pairs in Sublist $\mathrm{C}$ the $\mathrm{S}$ continues to give (most of the time) the items of higher frequency making the discrimination still easier. For Sublist I, however, the S typically responds with the less frequent of the two words. This reduces the differences in frequency between the two words and makes the old-new discrimination harder. Only a few exposures of the new correct items in Sublist I seems to make the discrimination more difficult; this is probably due to the fact that these are very high frequency words to begin with and so they are near the "familiarity ceiling."

\section{References}

Buchwald, A. M. Extinction after acquisition under different verbal reinforcement combinations. J. exp. Psychol., 1959, 57, 43-48. McClelland, D. C. Studies in serial verbal discrimination learning. II. Retention of responses to right and wrong words in a transfer situation. J. exp. Psychol., 1942, 31, 149-162.

Spence, J. T., Lair, C. V., \& Goodstein, L. D. Effects of different feedback conditions on verbal discrimination learning in schizophrenic and nonpsychiatric subjects. J. verbal Learn. verbal Behav., 1963. 2, 339-345.

Spence, J. T, Verbal discrimination performance under different verbal reinforcement combinations. J. exp. Psychol., 1964, 67, 195-197.

Spence, J. T. The effects of verbal reinforcement combination on the performance of a four-alternative discrimination task. $J$. verbal Learn. verbal Behav., in press.

Thomdike, E. L., \& Lorge, I. The teacher's word book of 30,000 words. New York: Columbia University Press, 1944.

Underwood, B. J., Jesse, F., \& Ekstr and, B. R. Knowledge of rights and wrongs in verbal-discrimination learning. $J$. verbal Learn. verbal Behav., 1964, 3, 183-186.

\section{Note}

1. I wish to express my thanks to Ronald Gordon and Daniel Goldberg for their assistance. A paper based on these data was read at the EPA convention in New York City, April, 1966. 